\title{
Análisis de textos pseudocientíficos desde las creencias epistemológicas de estudiantes de psicología
}

\author{
Analysis of pseudoscientific texts from the Psychology students' epistemic beliefs \\ José Manuel Meza Cano \\ Víctor Eduardo Salas García
}

\begin{abstract}
RESUMEN
En internet se encuentran diversos textos sobre pseudoterapias, pero que no cuentan con evidencia científica, es aquí en donde los psicólogos en formación deben contar con la capacidad para analizar las fuentes de conocimiento, lo que puede relacionarse con las creencias epistemológicas de cada estudiante. El objetivo del presente estudio fue analizar las argumentaciones de estudiantes de psicología al revisar textos de internet sobre pseudoterapias e identificar sus creencias epistemológicas. Es un estudio transversal, de caso, cuyo muestreo fue no-probabilístico por conveniencia, conformado por once estudiantes de psicología en la modalidad a distancia. Se les facilitó un guion con el cual debían analizar dos textos sobre pseudoterapias. Los resultados se obtuvieron mediante un análisis cuantitativo descriptivo y también de co-ocurrencias. Los hallazgos muestran que los estudiantes tienden a analizar la credibilidad de los textos a partir de las fuentes de información que citan, también se encontraron posicionamientos medianamente sofisticados respecto a la contrastación de fuentes y las nociones de certeza que provee la evidencia, aunque fueron pocos los que mostraron vínculos con el conocimiento previo de la disciplina. El guion mostró ser un andamio cognitivo que favorece en los estudiantes un nivel sofisticado de análisis de textos.
\end{abstract}

Palabras clave: andamio cognitivo, conocimiento, internet, pseudociencias, pseudoterapias.

\section{ABSTRACT}

The Internet is home to several texts on pseudo-therapies, but they do not have scientific evidence, and it is here where psychologists in training must have the ability to analyze the sources of knowledge, which can be related to the epistemological beliefs of each student. The aim of the present study was to analyze the arguments of psychology students when reviewing Internet texts on pseudo-therapies and to identify their epistemological beliefs. It is a cross-sectional, case study, whose sampling was non-probabilistic, by convenience, and consisted of eleven psychology students in the distance learning modality. They were given a script with which they had to analyze two texts on pseudo-therapies. The results were obtained by means of a descriptive quantitative analysis and also of co-occurrences. The findings show that the students tend to analyze the credibility of the texts based on the sources of information they cite, and they also found moderately sophisticated positions with respect to the contrast of sources and the notions of certainty provided by the evidence, although few of them showed links with previous knowledge of the discipline. The script proved to be a cognitive scaffold that favors a sophisticated level of text analysis in students.

Keywords: cognitive scaffold, knowledge, Internet, pseudosciences, pseudotherapies. 


\section{INTRODUCCIÓN}

$\mathrm{Al}$ realizar una búsqueda de prácticas más frecuentes de pseudoterapias en Google Académico en el periodo desde el 2016 a marzo del 2021, se obtiene que la palabra "reiki" arroja 11,500 resultados en todos los idiomas, mientras que al acotar la búsqueda en español se obtienen 1,360 resultados. Al emplear "Bach Flowers" arroja 165 resultados en todos los idiomas, en español el término "Flores de Bach" se incrementa a 454 resultados. ¿Cuál podría ser la razón de esto? Caballo y Salazar (2019) dan algunas pistas en relación con las Flores de Bach, puesto que al parecer en el año 1976 la Organización Mundial de la Salud (OMS) mencionó que podía ser visto como un sistema médico útil, o así fue interpretado, aunque en realidad se afirmó que es una práctica que no ocasiona efectos secundarios, pero no era un aval institucional.

Para el caso del reiki pueden encontrarse publicaciones con un aparente rigor científico, por ejemplo, McManus (2017) revisó la literatura disponible en inglés de este término, específicamente para estudios clínicos que comparan su efectividad con grupos control y con efectos placebo, encontrando que, de los 13 estudios revisados, ocho demostraron que el reiki fue más eficaz que el placebo, cuatro no tuvieron diferencias entre ambos grupos y uno favoreció al placebo. Este autor concluye que el reiki puede ser visto como una práctica terapéutica segura viable para la sanación del cuerpo y la mente. Esto es preocupante, pues parece una legitimación de prácticas de pseudoterapia que abre la puerta al resto de pseudociencias para ser vistas como alternativas confiables.

Murillo-Godínez (2019) llama la atención en este fenómeno, dado que los promotores de pseudociencia, pseudoterapias y pseudomedicina de vez en cuando llegan a visibilizarse en sitios web y revistas científicas, por ejemplo, un artículo que demostró el mecanismo por el cual actuaba la homeopatía y fue publicado en una revista científica, que luego fue refutado por la misma. Este mismo autor cita otros casos en que se han dado conferencias en escuelas mexicanas sobre prácticas pseudocientíficas, ejemplo de ello es una conferencia sobre "biomagnetismo médico" en

José Manuel Meza Cano. Profesor de tiempo completo en el Sistema de Universidad Abierta y Educación a Distancia de la FES Iztacala, México. Es miembro del Sistema Nacional de Investigadores, Nivel 1. Cuenta con estudios como licenciado en Psicología por la FES Iztacala y doctor en Psicología por la Facultad de Psicología, ambos de la UNAM. Realizó una estancia de investigación en el grupo de Tecnología Educativa de la Facultad de Ciencias de la Educación, Universidad de Sevilla, España. Es miembro evaluador externo de tesis del doctorado en Sistemas y Ambientes Educativos con sede en la Universidad Veracruzana. Correo electrónico: manuel.meza@iztacala.unam.mx. ID: https://orcid.org/0000-0002-9504-7906.

Víctor Eduardo Salas García. Asesor educativo en la Universidad Tecnológica Latinoamericana en Línea, México. Es licenciado en Psicología por la Facultad de Estudios Superiores Iztacala, perteneciente a la Universidad Nacional Autónoma de México. Ha desarrollado actividades relacionadas al diseño instruccional y espacios de aprendizaje. Cuenta con participaciones en proyectos académicos en Psicología SUAyED Iztacala y como conferencista en el encuentro Educatic del 2020. Correo electrónico: salasgvictorunam@gmail.com. ID: https://orcid.org/0000-0002-9061-4430. 
una importante universidad privada mexicana. Esto obtiene relevancia en el caso de los psicólogos en formación, quienes deben desarrollar habilidades para discernir y trasladar los resultados de la investigación científica a su ejercicio profesional, para así utilizar los tratamientos validados científicamente en bienestar del paciente. Bajo este contexto es importante conocer cómo es que los estudiantes en formación analizan la veracidad de textos sobre pseudoterapias.

\section{Prácticas basadas en evidencia en psicología}

En contraparte a las pseudoterapias se encuentran aquellas prácticas que tienen sus bases en la evidencia obtenida por la investigación científica; en el ámbito de la medicina se empezó a emplear la medicina basada en evidencia (MBE), entendida como la integración de la experiencia clínica, que se refiere a la competencia y el juicio obtenido a través de la práctica, y la evidencia clínica, refiriéndose a los resultados obtenidos de la investigación científica, puntualizando que ninguna por sí sola es suficiente para determinar el éxito de un tratamiento (Sackett, Rosenberg, Gray, Haynes y Richardson, 1996). La American Psychological Association (APA) tomó como referente la MBE y lo trasladó a la psicología, resultando en las prácticas basadas en evidencia en psicología (PBE), definidas como "la integración de la mejor investigación disponible y la experiencia resultado de la práctica clínica sin dejar de tomar en cuenta el contexto de las características culturales y las preferencias del paciente" (APA, 2006, p. 273).

Esto promueve y motiva a la práctica de una psicología eficaz como resultado del uso de la aplicación de principios de evaluación, de una relación terapéutica y de intervención empíricamente respaldados, aunado a un desarrollo continuo de pruebas con un enfoque en la mejora de la atención al paciente (APA, 2005). También es considerado como una ideología, debido a que responde a una razón ética que radica en proveer al paciente el mejor tratamiento posible, como una obligación ética para los profesionales en psicología y de otras ciencias, por lo que se considera negligente negarse a dar uso de tratamientos validados empíricamente (Moretti y Basler, 2016). Además, como lo menciona el código ético del psicólogo, se debe abstener de utilizar técnicas de evaluación e intervenciones que no estén sustentadas por una teoría y una metodología científica (artículo 44), y el trabajo del psicólogo debe estar fundamentado con evidencia científica (artículo 129) (Federación Nacional de Colegios, Sociedades y Asociaciones de Psicólogos de México, 2018).

Por lo tanto, en la formación del psicólogo deben promoverse aquellas habilidades de evaluación de la información, para que puedan trasladar y diseminar el conocimiento y las tecnologías derivadas de la investigación en psicología a las prácticas clínicas con el objetivo de garantizar la efectividad de sus intervenciones (Jiménez, 2016). Diversos estudios dan evidencia de la necesidad de promover el uso de prácticas y tratamientos basados en evidencia, ya que muchas veces estos llegan 
a ser desconocidos por los psicólogos (Gálvez, Corpas, Velasco y Moriana, 2019; Pérez, Contreras y Martínez, 2020).

\section{Pseudociencias}

Al hablar de pseudociencias se hace mención de una variedad de materias basadas en prácticas y creencias que se presumen como ciencias, pero no utilizan el método científico (Lifshitz, 2017). Martí-Sánchez y Roger-Monzó (2018) afirman que las pseudociencias hacen referencia a prácticas que carecen de respaldo de evidencias científicamente verificables, por lo tanto, se crea la impresión de que las prácticas se han realizado tomando en cuenta procedimientos con cierto rigor científico cómo lo son las PBE. Lifshitz (2017) menciona que hay pseudociencia que aparentemente sigue algunos pasos del método científico, pero que en realidad son estrategias mercadotécnicas para promover productos o servicios que pueden deslumbrar y que es posible que puedan aparecer en revistas de prestigio.

Domènech-Casal (2019) afirma que las pseudociencias presentan un énfasis en la confirmación, con ausencia de auto-evaluación y fundamentación dominada por testimonios de tipo “a mí me funcionó”, agregando a esto el uso de un lenguaje aparentemente sofisticado y científico que se puede encontrar en las PBE, que puede confundir al usuario final al emplear términos como "cuántico", "holístico", "resonancia", "energía" o "fluido", lo que puede dotarles de cierta credibilidad pero, como anteriormente se mencionó, la posible evidencia clínica por sí sola no es suficiente para determinar el éxito de un tratamiento (Sackett et al., 1996), menos sin el sustento científico.

Entre las características de las personas que creen con mayor frecuencia en las pseudociencias, Domènech-Casal (2019) menciona que son personas que justifican su posición a partir de las experiencias propias y de primera mano, con una tendencia a considerar menos creíbles los hechos científicos. Lifshitz (2017) afirma que estos adeptos basan su creencia en anécdotas que pueden tener su explicación en el efecto placebo o en la tendencia natural de ciertas enfermedades a curarse con el paso del tiempo. Por su parte, el surgimiento de las llamadas "terapias alternativas", que incluyen prácticas como la homeopatía, el biomagnetismo, la ozonoterapia o el reiki, generalmente son vistas como alternativas confiables por personas caracterizadas por contar con un pensamiento de tipo "mágico" (Domènech-Casal, 2019).

Además de ser integradas al imaginario individual, las pseudociencias han impregnado contextos como los medios de comunicación. Martí-Sánchez y Roger-Monzó (2018) realizaron una investigación en la prensa digital española a través del análisis del corpus de los contenidos sobre homeopatía en los tres principales diarios españoles, encontrando que el término homeopatía se relaciona con tres grandes ámbitos médico-farmacéuticos: ámbito académico, ámbito de las enfermedades y ámbito de las creencias. 
Cortiñas-Rovira y Zaragoza (2018) señalan que se le debe poner atención a la inclusión de las pseudociencias en las bases de datos asociadas a bibliotecas públicas digitales españolas puesto que puede dotar de cierta legitimidad a estas prácticas al estar incluidas en una institución social con prestigio académico, confundiendo al usuario final, dado que comparten espacio con contenido científico. Esto a su vez puede tener un efecto secundario aún más adverso, pues las pseudociencias podrían proveer de respuestas más simples que favorezcan la aparición de una brecha de conocimiento entre los que disponen de conocimientos especializados-científicos y las habilidades para diferenciarlos y aquellos que no cuentan con estos.

Cortiñas-Rovira y Zaragoza (2018) analizaron los catálogos en línea de 4,650 bibliotecas públicas para indagar sobre el número de documentos que incluyen en sus temas principales a las pseudociencias. Reportaron en sus hallazgos más de 22,000 títulos que abordan alguna temática pseudocientífica, que si bien es únicamente el $0.15 \%$ del total de títulos, lo consideraron preocupante.

Si bien el hecho de que las pseudociencias impacten el ámbito comunicativo y académico puede parecer poco peligroso, no es así cuando se involucran en el ámbito de la salud puesto que, como ya se mencionó anteriormente, las pseudociencias pueden tener repercusiones en las creencias de las personas sobre diferentes vías para atender su salud, además llegan a malversar la información sobre sus tratamientos empleando estudios en los que se demuestra su eficacia o efectividad, siendo que lo verdaderamente importante es conocer la eficiencia de un tratamiento, esto se obtiene al demostrar que se alcanzaron los objetivos clínicos con el menor costo posible (Moretti y Basler, 2016).

En la misma línea de la salud podemos incluir a la psicología y la psicoterapia, como ciencia y técnica; en este sentido, García y Gutiérrez (2020) afirman que existe una variedad de modelos psicoterapéuticos, pero no todos cuentan con un fundamento científico, por lo que sus resultados se enmarcan en una cuestión de fe o se trata del efecto placebo. Al respecto Caballo y Salazar (2019) enfatizan en los peligros de estas prácticas, al ser confundidas con terapia científica, dado que algunas personas pueden abandonar tratamientos eficaces sustituyéndolos por pseudoterapias como el reiki.

Desde la perspectiva de García y Gutiérrez (2020), se deben descartar aquellos métodos basados en el uso de energías no medibles e invisibles, para favorecer en su lugar los hechos científicos verificables y la evidencia, esto es lo que diferencia a la psicoterapia de los actos de fe. Al respecto Caballo y Salazar (2019) afirman que en el ámbito de la psicología clínica y la psicoterapia no se especula con energías misteriosas, ni es necesario recurrir a efectos mágicos para que las técnicas funcionen dado que las intervenciones son realizadas de manera sistemática, favoreciendo la publicación de los resultados en revistas científicas para que sean consultados y replicados. 
A la luz de estas problemáticas es imperativo dotar a los estudiantes de psicología de habilidades de análisis y una amplia formación en ciencia para que puedan tener claridad sobre el proceder de la psicología científica, promoviendo así el desarrollo de la capacidad de diferenciar la psicología de las pseudociencias que resultan principalmente en pseudoterapias. Domènech-Casal (2019) afirma que desde el nivel secundaria los estudiantes logran identificar más fácilmente los argumentos basados en datos que los basados en autoridad, por ejemplo, un docente; sin embargo, es necesario llevar a los estudiantes hacia un mayor sentimiento de pertenencia con la ciencia. Lo anterior es importante dado que autores como Lifshitz (2017) señalan que aquellos no cercanos a la ciencia muchas veces no cuentan con atributos cognitivos ni con habilidades metodológicas para discriminar lo científico de lo que no lo es. Uno de estos atributos cognitivos podría estar relacionado con las convicciones epistemológicas de los alumnos en las actividades científicas (Domènech-Casal, 2019), pues deben ver a la ciencia como algo que provee de evidencia, más allá de ser una transmisión autoritaria de conocimientos. Dada esta problemática, se considera importante hablar sobre las creencias epistemológicas como creencias acerca de la naturaleza del conocimiento y el proceso de conocer; esto, aplicado a las fuentes de información científica, puede perfilarse como una variable importante en la distinción entre ciencia y pseudociencia.

\section{Creencias epistemológicas}

Para poder referirnos a la validación del conocimiento, su naturaleza y el proceso de conocer se deben de tener en cuenta criterios de análisis para argumentar acerca de la veracidad de los textos y la evidencia que proporcionan; en este sentido, las creencias epistemológicas incluyen creencias acerca del conocimiento, cómo se construye, cómo se establecen los criterios para evaluarlo, en dónde reside el conocimiento y cómo ocurre el conocer, lo que modifica la comprensión del mundo mientras dota a las personas de herramientas para enfrentar la incertidumbre (Hofer y Pintrich, 1997).

Las investigaciones sobre creencias epistemológicas tienen como origen el estudio de Perry (1970), quien observó en estudiantes universitarios un desarrollo de algo que iba más allá de las estrategias académicas. Luego de entrevistarlos logró establecer nueve posiciones a partir de las cuales esquematizó cómo es que se relacionaban sus creencias sobre el aprendizaje, la autoridad, las fuentes del conocimiento y la identidad.

La primera posición fue denominada dualismo básico, en el cual la verdad y lo que la autoridad dicta son una misma cosa, por lo tanto, las nociones del bien y lo que la autoridad dice también son lo mismo y se verá como inválido lo que contradiga esta información. En las posiciones intermedias (2, 3 y 4) interviene la multiplicidad, en la cual se descubre la diversidad y la sensación de amplitud, aparece la sensación de incertidumbre, por lo que el individuo debe integrar los cambios y contar con 
habilidades de análisis para evitar sentirse demasiado confundido. Las posiciones $5 \mathrm{y}$ 6 hacen referencia al relativismo y el paso al compromiso, las ideas absolutas o dualistas se vuelven casos aislados y son interpretados bajo un contexto. Aparece el compromiso como el acto de un individuo agente y elector de aspectos en su vida, una afirmación dentro de un mundo relativista, apropiándose de sus decisiones, encontrando lo que quiere "ser". En las posiciones 7 a 9 se continúa el desarrollo del compromiso, el individuo se da cuenta del impacto del compromiso tomado, siendo responsable del papel que tendrá en su vida y de las acciones que lo llevarán a tomar caminos para ser quien ha decidido ser, buscando escenarios propicios para lograrlo, llegando a una madurez relacionada con la experiencia a partir de lo que el individuo considera que "es".

A partir del modelo evolutivo de Perry (1970) surgieron propuestas que consideraron a las creencias epistemológicas como multidimensionales; uno de los más aceptados es el de Hofer y Pintrich (1997), quienes propusieron un modelo que incluye la certeza del conocimiento, el conocimiento simple, el origen del conocimiento y la justificación de este (figura 1).

En el área sobre naturaleza del conocimiento se aborda la pregunta “¿qué es el conocimiento?", con dos dimensiones: la certeza del conocimiento se refiere al grado en el que se ve el conocimiento como fijo por un lado, o más fluido por otro; en niveles ingenuos se tiene la creencia de que existe una verdad mientras que en niveles más sofisticados el conocimiento evoluciona. La segunda dimensión de esta área se refiere a la simplicidad, por un lado, en el extremo ingenuo se ve al conocimiento como una acumulación de hechos aislados, conocibles y concretos, mientras que en la visión sofisticada se considera como conceptos interrelacionados, relativos y contextuales.

Del lado derecho del modelo se encuentra el área del proceso del conocer que responde a la pregunta “¿cómo se conoce?”, en ella se encuentra la fuente del conocimiento, en la cual en los niveles ingenuos se cree que el conocimiento se encuentra fuera del ser, en autoridades externas, por lo que debe ser transmitido por ellas, mientras que en la posición sofisticada se tiene la creencia de que el conocimiento puede ser construido en interacción con otros y a través de la reflexión. La última dimensión es la justificación del conocimiento, en la que se hace referencia a la evaluación de las demandas del conocimiento; al respecto los individuos ingenuos justifican sus creencias a través de la observación, lo que transmite autoridad, o sobre la base de lo que es correcto, mientras que en los niveles sofisticados se emplean estrategias de evaluación, indagación e investigación.

Muis, Chevrier y Singh (2018) afirman que este tipo de creencias tienen impacto en las actividades académicas de la mano de procesos como la autorregulación; por lo tanto influyen en la selección de estrategias metacognitivas, la identificación y evaluación de información pertinente para el aprendizaje, el establecimiento de los criterios 


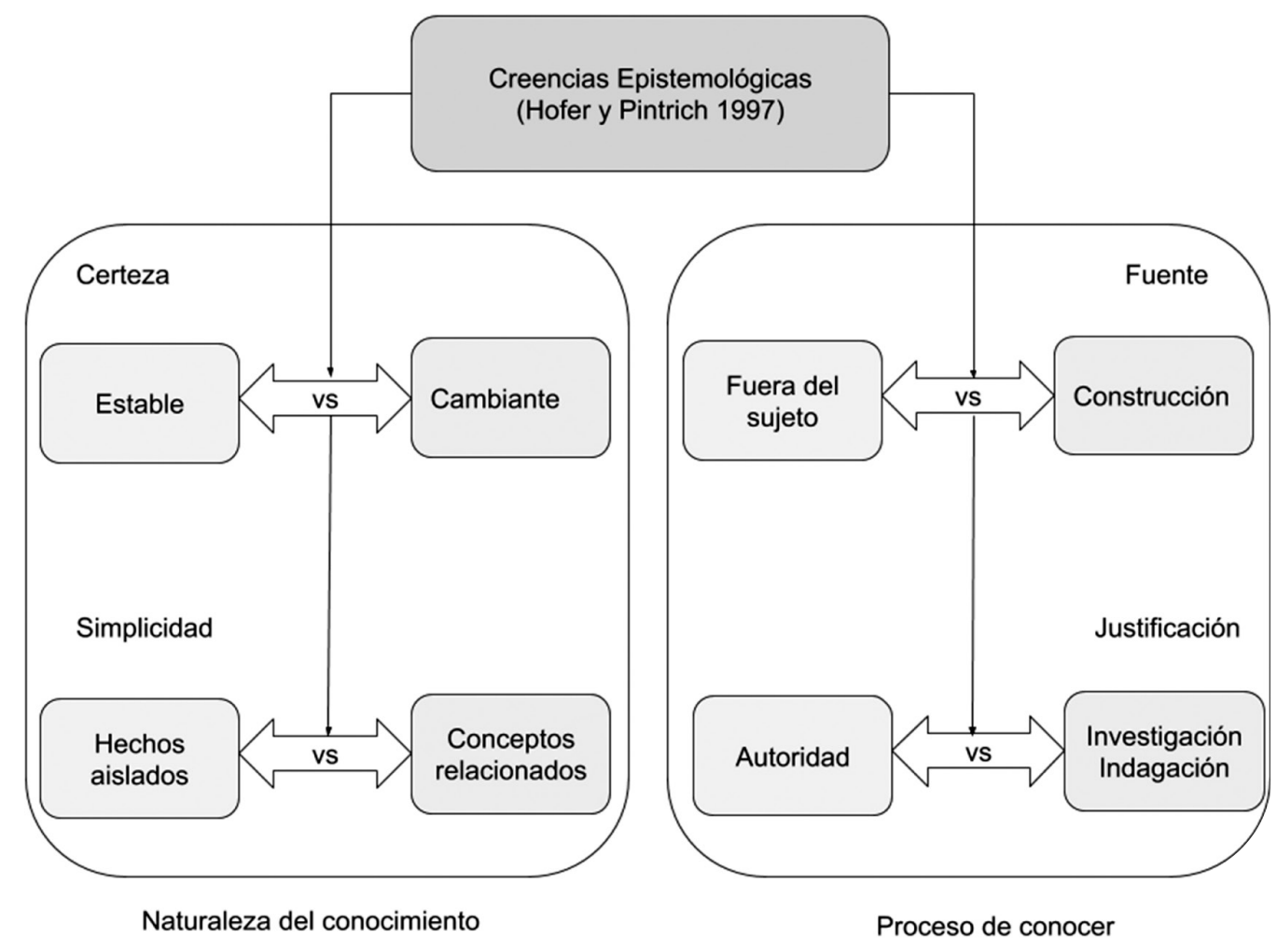

Figura 1. Muestra las dimensiones del modelo.

Fuente: Elaboración propia a partir del modelo de Hofer y Pintrich (1997).

y la comparación del avance con respecto a estos criterios de los productos realizados en un ciclo continuo de reflexión. A este respecto, si las creencias epistemológicas impactan en el aprendizaje y los procesos cognitivos relacionados con él, también podrían asociarse con el aprendizaje de la ciencia, dado que Zanotto y Gaeta (2018) afirman que es necesario el desarrollo de cuestionamientos, problematizaciones y análisis de información para obtener conocimiento científico y establecer diferencias con el uso de términos cotidianos, la apropiación y uso de teorías, y el desarrollo de estructuras conceptuales coherentes con la metodología de la investigación. Esto fue confirmado por investigaciones como la de Nussbaum, Sinatra y Poliquin (2008), quienes encontraron evidencia de que el posicionamiento epistemológico de estudiantes de nivel licenciatura se relacionó con el nivel de análisis crítico de problemas científicos, así como una diferencia en la interacción con los pares: aquellos estudiantes que se encontraron en posiciones de creencias intermedias fueron menos críticos con las inconsistencias y errores de los problemas científicos, en tanto que interactuaron menos con sus pares, mientras que los estudiantes con creencias más sofisticadas fueron más críticos, plantearon ideas variadas con sus pares y resolvieron problemas científicos con mayor precisión. 
El modelo de Hofer y Pintrich (1997) fue empleado por Braten, Stromso y Samuelsen (2005) situando la naturaleza del conocimiento y la forma de conocer en internet, denominando a este campo Creencias epistemológicas específicas a internet. En su estudio desarrollaron un instrumento que permitió posicionar a los estudiantes de acuerdo con sus creencias sobre el conocimiento que está en internet y sobre cómo es que se llega a conocer lo que está en internet de manera específica, encontrando que los estudiantes que creen que internet es una fuente de hechos simples, específicos, que contiene la verdad absoluta de las cosas, tienen menor disposición a manejar información que se contrapone, evaluarla y emplearla; en consecuencia, tratan al proceso de búsqueda en internet como algo sencillo, con exceso de confianza, y emplean los primeros resultados de búsqueda sin una evaluación exhaustiva.

Sobre esta línea de trabajo, Chiu, Liang y Tsai (2016) exploraron el papel de la experiencia previa en el uso de buscadores de internet y su relación con las creencias epistemológicas de estudiantes de entre 12 y 26 años. Sus hallazgos mostraron que aquellos participantes con una mayor cantidad de años de educación tendían a tener creencias sofisticadas acerca del conocimiento basado en internet, derivado de esto, tendieron a creer que internet es una fuente que contiene conocimientos precisos, además de justificar a través de estrategias de indagación el conocimiento basado en internet comparándolo con otras fuentes.

En el estudio de Kammerer, Braten, Gerjets y Stromso (2013) solicitaron a estudiantes que construyeran argumentaciones acerca de dos tipos distintos de terapias para la cura de una enfermedad poco común y sugerir un tratamiento para un amigo ficticio, para ello emplearon una página web que incluía enlaces a sitios con información contradictoria. Encontraron que cuando los estudiantes tienen creencias ingenuas sobre la certeza y la estructura del conocimiento por lo general no evalúan las fuentes en una tarea de búsqueda en internet ni realizan alguna verificación, tampoco realizan análisis de la información o uso del conocimiento previo, por lo que las argumentaciones sobre sus elecciones son pobres. Un hallazgo importante es que aquellos que le confieren a internet una excesiva confianza como fuente de consulta, con información precisa y hechos detallados, inspeccionan menos los enlaces del final de una lista de resultados dentro de un motor de búsqueda, lo que se alinea con los hallazgos de Tu, Shin y Tsa (2008), quienes encontraron que los estudiantes con creencias epistemológicas sofisticadas aplican estrategias para elegir, verificar y juzgar la información previo al desarrollo de una tarea académica.

Un caso con población mexicana, en el nivel de licenciatura, es el trabajo de Olvera (2017), en el cual empleando el modelo de Creencias epistemológicas específicas a internet evaluó a estudiantes que iniciaban su formación académica, a estudiantes que se encontraron en semestres intermedios y a estudiantes a punto del egreso, encontrando que aquellos que tenían estudios universitarios previos fueron quienes 
demostraron posicionamientos sofisticados, sobre todo con respecto a la creencia de que es el aprendiz quien construye el conocimiento, dejando de lado variables como el avance en el programa académico de dicha licenciatura, el género y la edad. Asimismo encontró en la muestra niveles medios de conformidad con el conocimiento de internet, por lo que tienen una alta tendencia a emplear estrategias de razonamiento y comparación de las fuentes, como personas, experiencias, documentos, etc., tomando el conocimiento como algo progresivo y que puede cambiar con el tiempo.

Con base en estas evidencias, cabe preguntarse cómo es que los estudiantes evalúan el conocimiento de fuentes provenientes de internet, cuáles son los niveles de certeza que le proveen a estas fuentes, cómo es que justifican el conocimiento y si son conceptualizadas como simples o como conceptos que se interrelacionan con otros, rescatando el conocimiento previo, especialmente al enfrentarse a documentos con contenido pseudocientífico, esto al ser parte de una tarea académica auténtica que, a decir de Shah (2015), involucra motivacionalmente a los participantes en la argumentación, por lo que deja entrever su posición epistemológica.

Dado lo anterior, el objetivo del presente estudio es analizar las argumentaciones de estudiantes de psicología al revisar textos de internet sobre pseudoterapias e identificar sus creencias epistemológicas.

\section{Metodología}

Se trata de un diseño transversal, un estudio de caso, puesto que se analiza a un grupo de once estudiantes de psicología en un único momento.

\section{Muestra}

Se trató de una muestra no probabilística por conveniencia, pues estuvo conformada por once estudiantes pertenecientes a los últimos dos semestres (octavo y noveno) de psicología pertenecientes a un programa en la modalidad a distancia, diez mujeres y un hombre, con una media de edad de 30 años.

\section{Escenario}

La actividad se enmarcó en un curso en línea de psicología como parte de las entregas a realizar para su aprobación, por lo que contó con un peso para la calificación final. Se tuvo un periodo de una semana para el desarrollo de la actividad.

\section{Instrumento}

Se compartió en la plataforma LMS Moodle el "Guion para el análisis de textos desde las creencias epistemológicas", el cual contaba con dos secciones, en una se debían llenar los espacios con la información general del texto a revisar y en la segunda se debía responder a preguntas abiertas sobre cada dimensión del modelo de creencias 
epistemológicas y cerrar con una conclusión sobre el texto. Se decidió añadir un apartado de "importancia" para verificar la argumentación de los estudiantes sobre la validez de los artículos para el campo de estudio, por lo que se tuvo un total de seis apartados. El instrumento se muestra en la tabla 1.

Tabla 1. Guion para el análisis de textos desde las creencias epistemológicas.

Tema principal:

$\begin{array}{cc}\begin{array}{l}\text { Revista/portal } \\ \text { (incluyendo su }\end{array} & \begin{array}{c}\text { Institución que } \\ \text { respalda (incluyendo } \\ \text { página web): }\end{array} \\ \text { su página web): }\end{array}$

Referencia APA:

Certeza: ¿Qué tan verdadero crees que es el conocimiento del texto? Argumenta la razón

Simplicidad/complejidad: ¿el conocimiento del texto se relaciona con otras disciplinas, conceptos o hechos?

Valoración de la fuente: ¿el conocimiento vertido es contrastable con otras fuentes como libros, internet o experiencia personal?

Importancia: menciona la importancia que podría tener el texto para el campo de estudio, si no la tiene argumenta la razón

Justificación: describe cómo haces para saber que el conocimiento vertido en el texto es verdadero o falso

Conclusión acerca del conocimiento expuesto en el texto

Fuente: Construcción personal.

\section{Procedimiento}

A los estudiantes se les facilitaron dos textos a analizar, uno de ellos hablaba del reiki para el tratamiento del estrés y la ansiedad (Kurebayashi, Turrini, Souza, Takiguchi, Kuba y Nagumo, 2016) y otro texto sobre Flores de Bach y su uso durante el embarazo (Blay, 2013). Algo importante de señalar es que dichos textos se encontraban publicados en sitios académicos: en una memoria de congreso y en una revista indizada por un buscador de renombre.

Se les facilitó el "Guion para el análisis de textos desde las creencias epistemológicas" y los estudiantes tuvieron una semana para realizar el trabajo y enviarlo a través de la plataforma Moodle en un espacio de tarea.

Posterior a esto se obtuvieron los guiones con sus respuestas, los cuales se analizaron empleando el software QDA Miner versión 6.0, definiendo los códigos a partir de lo encontrado en las respuestas de cada guion, enmarcando cada código en las categorías generales del modelo de Hofer y Pintrich (1997).

Después de la primera codificación, se compartieron los segmentos codificados a un segundo juez con quien se discutió la pertinencia de estos. Se realizaron ajustes a los códigos y se realizó una segunda codificación tomando en cuenta las sugerencias del segundo juez. 
Una vez obtenidos los segmentos codificados, se procedió a realizar un análisis cuantitativo descriptivo y también de co-ocurrencias empleando el coeficiente de Jaccard, el cual es un índice calculado a partir de datos cualitativos empleado para el análisis de la biodiversidad en un ambiente dado; el coeficiente puede tomar valores entre 0 (cuando no hay especies compartidas entre dos sitios) hasta 1 (cuando los dos sitios tienen la misma composición de especies) (Moreno, 2011; Hodges y McKinney, 2018). Se ha empleado en otros contextos fuera de la biología para analizar las co-ocurrencias de dos fenómenos, por ejemplo, Majumder, Pakray, Gelbukh y Pinto (2016) lo emplearon para realizar mediciones de similaridad semántica entre oraciones a partir del resumen de documentos; Gillani y Eynon (2014) lo emplearon para el análisis de la actividad de los foros de discusión de un curso masivo abierto en línea (MOOC), mientras que Rauchfleisch (2017) empleó dicho índice para analizar las publicaciones y las citas de artículos de un tema específico.

\section{REsultados}

A partir de la codificación se obtuvo en total 173 segmentos correspondientes a cada uno de los seis apartados del guion, en un total de 27 códigos, los cuales se muestran en la tabla 2.

Como puede notarse, el código con mayor frecuencia de aparición fue Sin respaldo de fuentes, perteneciente al apartado de Fuente del conocimiento, con 17 segmentos codificados, lo que corresponde con el 9.8\% del total de los códigos y apareciendo en 9 de los 11 casos, seguido de Contrastación/indagación con otras fuentes correspondiente al apartado de Justificación, con 15 segmentos codificados, lo que representa el 8.7\% del total de los códigos y apareciendo en 8 de los 11 casos. Los códigos con menor frecuencia de aparición fueron Contrastable con experiencia ingenua dentro del apartado de Fuente del conocimiento y Sugerencias metodológicas dentro del apartado de Conclusiones, ambos con una única aparición, lo que representa el 0.6\% y en un único caso.

A pesar de que la información descriptiva a partir de la frecuencia de los códigos permite indagar sobre el tipo de argumentaciones más frecuentes por segmento tomado como categoría, se procedió a realizar un análisis de co-ocurrencias por cada caso, tomando como caso cada uno de los guiones analizados. Una co-ocurrencia sucede cuando dos códigos aparecen simultáneamente en un mismo caso, generando así pares de códigos y la frecuencia con la que coincidieron en el total de guiones codificados. Esto se presenta la figura 2, en donde puede notarse que los códigos Sin respaldo de fuentes (categoría: Fuente) e Información sin evidencia (categoría: Certeza) co-ocurrieron 7 veces; Sin respaldo de fuentes (categoría: Fuente) y Si no es científico no es verdad (categoría: Certeza) fueron las de mayor frecuencia de co-ocurrencia, seguida de otras co-ocurrencias con una frecuencia de 6 como son los pares: Falta de evidencias científicas (categoría: Importancia)-Contrastación/indagación (categoría: Justificación), Infor- 
Tabla 2. Categorías, códigos, frecuencias y casos en los que los códigos tuvieron presencia.

\begin{tabular}{|c|c|c|c|c|c|}
\hline Categoría & Código & Definición & $\begin{array}{l}\text { Fre- } \\
\text { cuencia }\end{array}$ & $\begin{array}{l}\% \text { de } \\
\text { códigos }\end{array}$ & Casos \\
\hline Certeza & Si no es científico no es verdad & $\begin{array}{l}\text { Considera que el conocimiento del texto no tiene bases científicas, } \\
\text { por lo tanto no es cierto }\end{array}$ & 12 & $6.9 \%$ & 9 \\
\hline Certeza & Verdad y conocimiento previo & Considera lo que es verdadero a partir de lo que sabe del tema & 9 & $5.2 \%$ & 3 \\
\hline Certeza & Información sin evidencia & $\begin{array}{l}\text { El texto muestra información contradictoria y carente de evidencia, } \\
\text { que confunde }\end{array}$ & 14 & $8.1 \%$ & 8 \\
\hline Simplicidad & Otras prácticas similares & $\begin{array}{l}\text { Se mencionan prácticas similares bajo el mismo enfoque no } \\
\text { científico }\end{array}$ & 6 & $3.5 \%$ & 6 \\
\hline Simplicidad & No conexión con psicología & No hay una conexión de lo expuesto con la psicología & 8 & $4.6 \%$ & 5 \\
\hline Simplicidad & No relación científica & No hay relación entre lo expuesto y las disciplinas científicas & 9 & $5.2 \%$ & 7 \\
\hline Simplicidad & Conexión con la psicología & Se menciona que el texto tiene conexión con la psicología & 3 & $1.7 \%$ & 3 \\
\hline Simplicidad & $\begin{array}{l}\text { Conexión con disciplinas } \\
\text { científicas }\end{array}$ & Se relaciona y acepta en disciplinas científicas & 2 & $1.2 \%$ & 2 \\
\hline Fuente & Sin respaldo de fuentes & Afirmaciones del texto que no tienen ningún respaldo en fuentes & 17 & $9.8 \%$ & 9 \\
\hline Fuente & Fuentes autorreforzantes & Las fuentes empleadas refuerzan lo presentado en el texto & 8 & $4.6 \%$ & 7 \\
\hline Fuente & Lenguaje poco válido & El lenguaje empleado le quita validez al texto & 3 & $1.7 \%$ & 2 \\
\hline Fuente & Bibliografía no confiable & La bibliografía presentada no es confiable & 3 & $1.7 \%$ & 2 \\
\hline Fuente & $\begin{array}{l}\text { Fuentes contrarrestan } \\
\text { información }\end{array}$ & Incluye fuentes que contrarrestan la información del texto & 3 & $1.70 \%$ & 1 \\
\hline Fuente & $\begin{array}{l}\text { Contrastable con experiencia } \\
\text { ingenua }\end{array}$ & $\begin{array}{l}\text { El conocimiento sólo es contrastable con experiencias de personas } \\
\text { que han utilizado estas terapias o que no cuentan con conocimiento } \\
\text { científico-psicológico }\end{array}$ & 1 & $0.6 \%$ & 1 \\
\hline Importancia & Falta de evidencias científicas & El texto carece de evidencias científicas, por lo que no es importante & 14 & $8.1 \%$ & 8 \\
\hline Importancia & $\begin{array}{l}\text { Falta de referencias resta } \\
\text { importancia }\end{array}$ & Pierde importancia al no mencionar a otros autores & 3 & $1.7 \%$ & 3 \\
\hline Importancia & Aparente importancia & $\begin{array}{l}\text { Por la forma en la que está expresado parece importante pero } \\
\text { no tiene sustento, intenta persuadir }\end{array}$ & 7 & $4.0 \%$ & 5 \\
\hline Importancia & Falta de rigor metodológico & $\begin{array}{l}\text { El texto no explica o no contiene suficiente conocimiento } \\
\text { metodológico para ser tomado en cuenta }\end{array}$ & 5 & $2.9 \%$ & 3 \\
\hline Justificación & $\begin{array}{l}\text { Contrastación con } \\
\text { conocimiento previo }\end{array}$ & Contrastación con conocimiento previo del tema & 5 & $2.9 \%$ & 5 \\
\hline Justificación & $\begin{array}{l}\text { Contrastación/indagación } \\
\text { con otras fuentes }\end{array}$ & $\begin{array}{l}\text { Contrasta el conocimiento con otras fuentes que considera válidas. } \\
\text { Revisa las fuentes presentadas en el texto o indaga en el tema }\end{array}$ & 15 & $8.7 \%$ & 8 \\
\hline Justificación & $\begin{array}{l}\text { No creíble, falta de fuentes } \\
\text { científicas }\end{array}$ & $\begin{array}{l}\text { Justifica la poca credibilidad con base en la carencia de fuentes } \\
\text { científicas }\end{array}$ & 14 & $8.1 \%$ & 7 \\
\hline Justificación & $\begin{array}{l}\text { Falsedad por ser } \\
\text { autorreforzante }\end{array}$ & $\begin{array}{l}\text { El texto emplea fuentes autorreforzantes, por tanto tiene poca } \\
\text { credibilidad }\end{array}$ & 2 & $1.2 \%$ & 2 \\
\hline Justificación & $\begin{array}{l}\text { Congruencia } \\
\text { teórico-metodológica }\end{array}$ & $\begin{array}{l}\text { Identifica que hay congruencia de los conceptos con el método } \\
\text { empleado para su investigación }\end{array}$ & 2 & $1.2 \%$ & 2 \\
\hline Justificación & $\begin{array}{l}\text { Requiere más pruebas } \\
\text { empíricas }\end{array}$ & $\begin{array}{l}\text { Se requieren de mayor información empírica para decir que el } \\
\text { conocimiento expresado es plausible }\end{array}$ & 3 & $1.7 \%$ & 2 \\
\hline Conclusión & Publicado pero no científico & Aunque el texto está publicado, no quiere decir que sea científico & 2 & $1.2 \%$ & 2 \\
\hline Conclusión & Métodos no válidos & No contiene métodos congruentes desde el punto de vista científico & 2 & $1.2 \%$ & 2 \\
\hline Conclusión & Sugerencias metodológicas & $\begin{array}{l}\text { El lector agrega sugerencias que pueden aportar credibilidad } \\
\text { al conocimiento del texto }\end{array}$ & 1 & $0.6 \%$ & 1 \\
\hline
\end{tabular}

Fuente: Construcción personal.

mación sin evidencia (categoría: Certeza)-Contrastación/Indagación (categoría: Justificación) y otros. Cabe mencionar que estas co-ocurrencias se presentan sin tomar en cuenta el apartado del guion en el que se encuentren, tomando cada caso en su conjunto. 


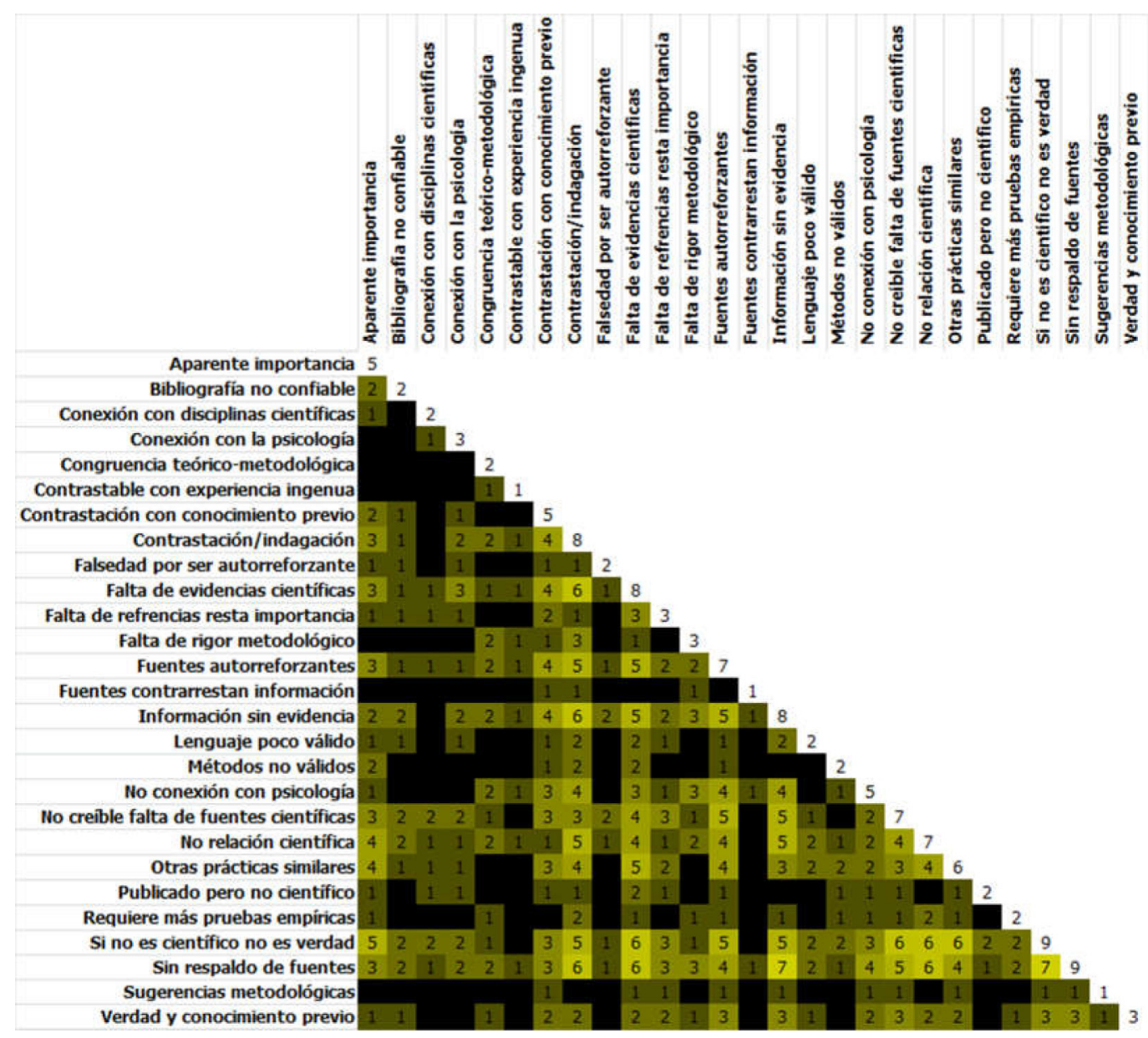

Figura 2. Frecuencia de co-ocurrencia de códigos por cada caso.

Fuente: Construcción personal.

Siguiendo la misma lógica, existieron pares de códigos que no co-ocurrieron ni una vez en los casos analizados, por ejemplo, Conexión con disciplinas científicas (categoría: Simplicidad)-Bibliografía no confiable (categoría: Fuente), por tanto, en la figura 2 aparecen con recuadros negros, vacíos.

A partir de las co-ocurrencias se generó el análisis de conglomerados empleando el índice de Jaccard, el cual se muestra en la figura 3.

El análisis de conglomerados permite visualizar cómo es que los códigos se agrupan de acuerdo con sus co-ocurrencias. Debajo se muestra una escala que va de 1.0 a 0.0, lo que indica que, entre más cercano a la izquierda se encuentre esta relación, es más probable que estos códigos aparezcan juntos en un caso. Como se puede notar se crearon seis conglomerados, los cuales se presentan con diferentes colores. Se visualiza un primer conglomerado de color azul oscuro el cual incluye 16 de los 27 códigos, seguido de un segundo conglomerado que incluye cuatro códigos en color amarillo. El resto de los conglomerados tiene solo dos códigos y un código apareció sin una relación con el resto de los códigos: Fuentes contrarrestan información. 

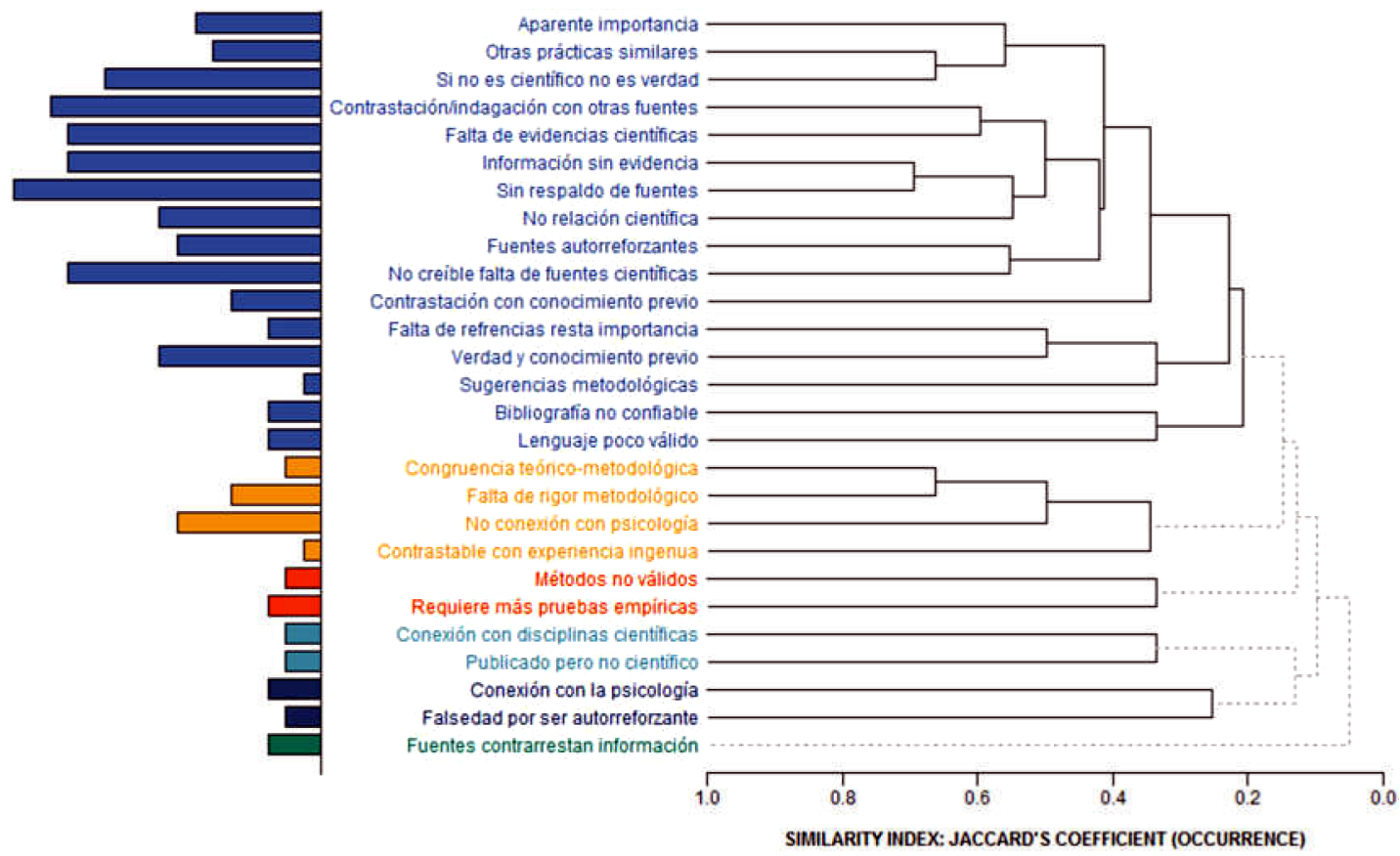

Figura 3. Análisis de conglomerados empleando el índice de Jaccard.

Fuente: Construcción personal.

Tabla 3. Muestra los códigos con coeficientes de Jaccard igual y mayores a 0.60.

Código 1

Código 2

Índice de similaridad

\begin{tabular}{ccc}
\hline Información sin evidencia & Sin respaldo de fuentes & 0.70 \\
\hline Otras prácticas similares & Si no es científico no es verdad & 0.66 \\
\hline Congruencia teórico-metodológica & Falta de rigor metodológico & 0.66 \\
\hline Contrastación/indagación con otras fuentes & Falta de evidencias científicas & 0.60 \\
\hline
\end{tabular}

Fuente: Construcción personal.

Para mostrar los coeficientes derivados del índice de Jaccard de acuerdo con los pares de códigos se obtuvieron los datos de la tabla 3.

Puede observarse que fueron cuatro los pares de códigos con un índice de Jaccard mayor a 0.60, siendo el par Información sin evidencia (categoría: Certeza) y Sin respaldo de fuentes (categoría: Fuente) el que alcanzó un índice de Jaccard de 0.70. El resto de los pares, si bien mostraron índices positivos, fueron menores a 0.69.

\section{Conclusiones}

El objetivo de este trabajo fue analizar las argumentaciones de estudiantes de psicología al revisar textos de internet sobre pseudoterapias e identificar sus creencias epistemológicas. Las conclusiones versan sobre tres ejes principales: los resultados descriptivos sobre la frecuencia de los códigos que surgieron a partir de las argu- 
mentaciones de los participantes en cada segmento del guion, luego el análisis de las co-ocurrencias de estos códigos y por último la pertinencia del guion como andamio cognitivo que favorece el análisis del conocimiento que se encuentra en fuentes de internet, así como las limitaciones del estudio y líneas de investigación futuras.

Los hallazgos muestran una alta tendencia a analizar las fuentes de información como una vía para dotar de credibilidad al conocimiento de los textos, es por esta razón que el código Sin respaldo de fuentes obtuvo la mayor frecuencia de aparición, en la mayoría de los casos, así como el código Contrastación/indagación con otras fuentes. Esto se relaciona con las estrategias de análisis de los participantes a quienes se les podría colocar con un posicionamiento epistemológico medio-sofisticado en las categorías de Fuente y Justificación del conocimiento de acuerdo con el modelo de Hofer y Pintrich (1997), dado que tienden a analizar las fuentes de información para dotar o no de valor al conocimiento del texto. A pesar de esto, hay un código que hizo aparición en pocos casos, denominado Contrastación con conocimiento previo, y que hace referencia a un proceso reflexivo de recuperación de conocimiento que se tenía anteriormente sobre el tema, lo cual llevaría a afirmar que estos cinco casos representan a participantes con un posicionamiento altamente sofisticado en la categoría de la Justificación del conocimiento pues, a decir de Zanotto y Gaeta (2018), confían en su propio razonamiento, en la experiencia previa y en el uso de normas de investigación e integración de fuentes de información, por lo que han dejado de lado creencias sobre la autoridad como única fuente de conocimiento.

Por otro lado, el análisis de la evidencia mostrada en los textos es un elemento que se posicionó como algo primordial dentro de la muestra, siendo el código Información sin evidencia, dentro de la categoría Certeza del conocimiento, uno de los que obtuvo mayor frecuencia, en 8 de los 11 casos. Esto posiciona a la mayoría de los participantes con una tendencia sofisticada en esta categoría puesto que, como afirmó Perry (1970), el proceso de análisis de la evidencia es una muestra de un posicionamiento relativista y contextual, en el cual el conocimiento adquiere valor de manera situada, por tanto, las nociones de "verdad" que podría tener un texto que habla de reiki o Flores de Bach en un contexto académico, como lo sería una revista indizada o una memoria de congreso, les pareció fuera de lugar. De la misma manera, el código de Verdady conocimiento previo, que apareció únicamente en 3 de los 11 casos, muestra un posicionamiento altamente sofisticado, por lo que involucra la recuperación, reflexión y contrastación del conocimiento del texto con el propio para emitir así un juicio, lo que denota una alta capacidad de análisis y nociones de certeza relativistas.

Es importante llamar la atención hacia aquellos códigos con menos frecuencia, pues son ejemplos de argumentaciones poco comunes; en ellos se encuentran principalmente dos: Contrastable con experiencia ingenua dentro de la categoría de Fuente del conocimiento, que hace referencia a que el conocimiento solo puede discutirse si se 
posiciona desde el mismo tipo de área pseudocientífica, la cual ven los estudiantes como ajena, distante de la psicología como ciencia; y de manera similar con el código Sugerencias metodológicas dentro del apartado de Conclusiones, en donde los estudiantes hacen referencia a procedimientos alternativos que pueden seguirse empleando métodos basados en evidencia para abordar los problemas planteados. En estos dos casos se puede afirmar que son códigos con un alto grado de sofisticación, puesto que son reflexiones más allá de solo descartar la información, dado que se ofrecen ejemplos de formas de proceder desde la psicología científica que los estudiantes han aprendido a partir de su trayectoria escolar. Esta diferencia es descrita también por Bontempo y Flores (2016), que señalan que los estudiantes pasan de posiciones ingenuas en las cuales creen que la realidad puede ser conocida como tal (de primera mano) hacia posiciones sofisticadas en las que se reconoce la necesidad de relativizar el conocimiento con evidencias vinculadas a un contexto, basando su seguridad en conocimientos que perciben en sí mismos.

A partir del análisis de co-ocurrencias y del índice de Jaccard se encontraron principalmente hallazgos de cómo es que dos códigos aparecen de manera simultánea en los guiones de los estudiantes. Era hasta cierto punto esperable que estos pares se conjuntaran de manera lógica en razón del tipo de estrategias y procedimientos que demostraron en sus argumentaciones, siendo Sin respaldo de fuentes (categoría: Fuente) e Información sin evidencia (categoría: Certeza) en los que es claro el peso que los participantes dieron al papel de las fuentes y su relación con la evidencia científica, a pesar de que se trate de dos dimensiones distintas del modelo de Hofer y Pintrich (1997).

Un hallazgo importante es que la muestra reconoció prácticas pseudocientíficas al mencionar que solo son congruentes con prácticas similares, agrupándolas como "no-científicas", a través del código Otras prácticas similares (categoría: Simplicidad) que a su vez se relacionó con afirmaciones en las que cuestionaron la veracidad y certeza de la información sin bases científicas, esto en el código Si no es científico no es verdad (categoría: Certeza). Esto permite afirmar que la muestra tiene la capacidad de reconocer las prácticas, pero al mismo tiempo afirman que la ciencia provee de certezas. Esto es un primer paso que puede dar origen al cuestionamiento de lo que se ve aparentemente como científico, pero no lo es, fomentando la capacidad de análisis en favor del vínculo teoría-práctica científica (Zanotto y Gaeta, 2018).

Con respecto al análisis a partir de las co-ocurrencias y el índice de Jaccard se obtuvieron cuatro conglomerados que agrupan a los códigos de acuerdo con su aparición en los guiones en relaciones directas e indirectas. A partir de este análisis se proponen cuatro tipos de énfasis que pueden encontrarse en las argumentaciones de los participantes (ver tabla 4).

Como puede notarse, al primer conglomerado se le denominó "Énfasis en la veracidad del conocimiento a partir de la evidencia científica, las fuentes de información 
Además, que se encuentre evidencia de estas estrategias juntas puede tener diferentes resultados positivos, dado que Brandmo y Bråten (2018) afirman que en problemas científicos se observa que la justificación por múltiples fuentes puede favorecer el interés desarrollado en un tema, y esto a su vez puede generar la construcción de una base de conocimientos confiable.

El tercer conglomerado se denominó "Énfasis en el conocimiento metodológico y empírico de la ciencia”, en el cual, a pesar de estar compuesto por dos códigos, estos hacían menciones explícitas sobre la ciencia y los métodos con los que cuenta para validar el conocimiento, logrando ir más allá de la psicología como una ciencia basada en evidencia (Moretti y Basler, 2016), y tomando en cuenta la integración de los resultados de la investigación científica y la experiencia aplicada (APA, 2006; 2005).

El cuarto conglomerado, denominado "Énfasis en el análisis del conocimiento en las fuentes de información y su conexión con la psicología”, agrupó dos códigos que hacían hincapié en las fuentes de información empleadas en los textos y los textos de psicología comúnmente revisados, por lo que se mencionaron dudas sobre si estaba o no relacionado con las bases teóricas del ámbito psicológico.

Parece ser entonces que los dos últimos conglomerados se refieren a relaciones específicas entre teoría y método, algo importante sobre lo que Zanotto y Gaeta (2018) mencionan la existencia de una desarticulación en la enseñanza de la teoría y los métodos de investigación. Esta segmentación teoría-práctica puede derivar en la creencia ingenua sobre la justificación del conocimiento que da prioridad a la experiencia básica, de primera mano, lo que podría resultar en la idea de que la ciencia se construye a partir de experiencias simples (Zanotto y Gaeta (2018).

Hablando de internet como fuente de conocimiento, al parecer tuvo un impacto en las conclusiones obtenidas en dos de los casos analizados, puesto que hicieron referencia a que, aun siendo documentos publicados en sitios académicos (una revista científica y una memoria de congreso), eso no implica que el conocimiento de ambos textos obtenga con ello validez y por lo tanto fueran tomados como algo que dota de certeza. Por lo tanto, este hallazgo se alinea con el trabajo de Braten, Stromso y Samuelsen (2005), quienes afirman que aquellos estudiantes que creen que internet es una fuente que contiene hechos específicos, con verdades absolutas, no logran manejar la información que se contrapone, ni realizan evaluaciones sobre la misma. No fue el caso de la muestra del presente estudio, por tanto, son capaces de ver a internet como una fuente de conocimiento que no siempre es confiable.

Con respecto al uso del guion como un andamio cognitivo, puede afirmarse que fue un buen recurso dado que permitió realizar el análisis del conocimiento de un texto de internet, siendo eficiente para poder promover estas habilidades que permiten identificar prácticas basadas en evidencia de las que no (Gálvez et al., 2019; Pérez, Contreras y Martínez, 2020), y no solo eso, los llevó a que revisaran la información, 
la fuente de donde provenía, junto con el formato APA, la institución que lo respalda y su análisis de acuerdo con el modelo de Hofer y Pintrich (1997), invitando a que realizaran una conclusión de cierre sobre el texto.

El guion logró crear un escenario para que se pudieran emitir juicios en un problema que no tiene una única respuesta y requiere de argumentaciones, orientando las estrategias de análisis, recolección de información, interpretación y las formas de abordar los grados de certeza para tomar un posicionamiento (Terrazas, 2016). Este tipo de instrumentos han mostrado ser favorecedores como guías que motivan a los estudiantes a realizar actividades que demandan un nivel sofisticado de análisis de prácticas pseudocientíficas (Domènech-Casal, 2019), o también en el caso de solución de problemas que tienen poca estructura (Yun-Jo, 2010; Ojeda, 2011), por lo que el "Guion para el análisis de textos desde las creencias epistemológicas" parece favorecedor en este mismo sentido.

Conocer sobre las creencias epistemológicas permite que los estudiantes en formación sean capaces de identificar cómo es que ellos perciben el conocimiento que se les presenta y cuestionen la certeza que le otorgan a las fuentes que consultan (Braten, Stromso y Samuelsen, 2005); además permite que tanto docentes como estudiantes puedan determinar en qué nivel de sofisticación se encuentran para proceder a su mejora. Por lo tanto, sería pertinente ponerlo a disposición de más estudiantes en diferentes momentos de su desarrollo profesional, y así saber si cuentan con las habilidades suficientes para vincular problema, método y teoría (Jiménez, 2016). Esta variable psicológica puede aportar a un diagnóstico, pero también un eje de intervención para los psicólogos en formación de pregrado, dado a que el psicólogo es formado como investigador, por lo que prepararlos en una forma de pensamiento sofisticada de análisis y reflexión sobre el conocimiento sería una habilidad favorecedora para un profesional de la psicología o un aspirante a posgrado.

Entre las limitaciones del estudio se puede mencionar la cantidad de participantes, si bien son once personas, aun así, se obtuvo información relevante para analizar las argumentaciones con base en el modelo de Hofer y Pintrich (1997). Se pretende en un futuro aplicar el guion con una muestra mayor.

Entre los trabajos de investigación a futuro se plantea una intervención que permita promover posicionamientos epistemológicos sofisticados de los estudiantes. En la muestra se encontró evidencia de posicionamientos medios-sofisticados respecto a la contrastación de fuentes y la evaluación de la certeza que provee la evidencia, sin embargo, fueron menos los vínculos de estas argumentaciones con el conocimiento previo, por lo que se busca enfatizarlo (Olvera, 2017). Por lo anterior, se puede afirmar que en esta muestra se encuentran transitando a posiciones más sofisticadas en las cuales se dan cuenta de que son ellos quienes pueden construir su propio conocimiento a través de la reflexión y la interacción. Esto podría favorecer 
a los estudiantes a egresar con una preparación académica y de pensamiento suficiente para ingresar al posgrado, pues a decir de Zanotto y Gaeta (2018), el nivel de licenciatura generalmente se enfoca a la repetición de contenidos, siendo el posgrado el nivel ideal para favorecer la formación epistemológica de los estudiantes en un contexto de investigación y aprendizaje de la ciencia, por lo que cerrar esta brecha puede promover el egreso de estudiantes de licenciatura mejor preparados.

\section{Agradecimientos}

Este trabajo fue realizado gracias al proyecto PAPIIT-IA302121 Creencias Epistemológicas Específicas a Internet y su relación con la discriminación de noticias falsas en redes sociales.

\section{REFERENCIAS}

APA [American Psychological Association] (2005). Report of the 2005 presidential task force on evidencebased practice. Washington, DC: APA.

APA Presidential Task Force on Evidence-Based Practice (2006). Evidence-based practice in psychology. The American Psychologist, 61(4), 271-285.

Blay, E. (2013). Gestación, nacimiento y Flores de Bach. IV Congreso Sedibac de Terapia Floral. Recuperado de: https://sedibac.org/wp-content/ uploads/2018/01/24_Ponencia_Enrique_Blay. pdf.

Bontempo, L., y Flores, R. (2016). El desarrollo de la auto-autoría en estudiantes de Psicología de la UNAM. Psicología Iberoamericana, 24(1), 30-37.

Brandmo, C., y Bråten, I. (2018). Investigating relations between beliefs about justification for knowing, interest, and knowledge across two socio-scientific topics. Learning and Individual Differences, 62, 89-97. https://doi.org/10.1016/j. lindif.2018.01.010.

Braten, I., Stromso, H., y Samuelsen, M. (2005) The relationship between Internet-specific epistemological beliefs and learning with Internet technologies. Educational Computing Research, 33(2) 141-171.

Caballo, V., y Salazar, I. (2019). Ingenuos. El engaño de las terapias alternativas. España: Siglo XXI.

Chiu, Y., Liang, J., y Tsai, C. (2016). Exploring the roles of education and Internet search experience in students' Internet-specific epistemic beliefs.
Computers in Human Behavior, 62, 286-291. https://doi. org/10.1016/j.chb.2016.03.091.

Cortiñas-Rovira, S., y Zaragoza, M. (2018). Análisis de la presencia de pseudociencia en los catálogos de las bibliotecas públicas españolas. Revista Española de Documentación Científica, 41(1), 197. https://doi.org/10.3989/ redc.2018.1.1474.

Domènech-Casal, J. (2019). Escalas de certidumbre y balanzas de argumentos: una experiencia de construcción de marcos epistemológicos para el trabajo con pseudociencias en secundaria. Apice. Revista de Educación Científica, 3(2), 37-53. https://doi.org/10.17979/arec.2019.3.2.4930.

Federación Nacional de Colegios, Sociedades y Asociaciones de Psicólogos de México (2018). Código de ética de las y los psicólogos mexicanos (pp. 36 y 53).

Gálvez, M., Corpas, J., Velasco, J., y Moriana, J. (2019). El conocimiento y el uso en la práctica clínica de los tratamientos psicológicos basados en la evidencia. Clínica y Salud, 30(3), 115-122. https://dx.doi.org/10.5093/ clysa2019a12.

García, J., y Gutiérrez, M. (2020). De la pseudoterapia a la psicoterapia basada en evidencia, ¿por qué es importante diferenciarlas? Educación y Salud Boletin Cientifico Instituto de Ciencias de la Salud Universidad Autónoma del Estado de Hidalgo, 8(16), 65-68.

Gillani, N., y Eynon, R. (2014). Communication patterns in massively open online courses. The Internet and Higher Education, 23, 18-26. https://doi.org/10.1016/j.iheduc.2014.05.004. 
Hodges, M., y McKinney, M. (2018). Urbanization impacts on land snail community composition. Urban Ecosystems, 21(4), 721-735. https://doi.org/10.1007/ s11252-018-0746-x.

Hofer, B., y Pintrich, P. (1997). The development of epistemological theories: Beliefs about knowledge and knowing and their relation to learning. Review of Educational Research, 67(1), 88-140.

Jiménez P., A. (2016). Análisis multimétodo del proceso de cambio de habilidades metodológicas, conceptuales y de intervención en estudiantes de posgrado [Tesis de Doctorado]. Universidad Nacional Autónoma de México, Ciudad de México.

Kammerer, Y., Braten, I., Gerjets, P., y Stromso, H. (2013). The role of Internet-specific epistemic beliefs in laypersons' source evaluations and decisions during web search on a medical issue. Computers in Human Behavior, 29, 1193-1203.

Kurebayashi, L., Turrini, R., Souza, T., Takiguchi, R., Kuba, G., y Nagumo, M. (2016). Masaje y reiki para reducción de estrés y ansiedad: ensayo clínico aleatorizado. Revista Latino-Americana de Enfermagem, 24. Recuperado de: https://www.scielo.br/j/rlae/a/77V F53WysdCSsPmDXpSmzdb/?format=pdf\&lang=es.

Lifshitz, A. (2017). La pseudociencia y los falsos investigadores. Medicina Interna de México, 33(4), 439-441. Recuperado de: http://www.scielo.org.mx/scielo.php?script=sci_arttextypid $=$ S0186-48662017000400439.

Majumder, G., Pakray, P., Gelbukh, A., y Pinto, D. (2016). Semantic textual similarity methods, tools, and applications: A survey. Computación y Sistemas, 20(4). https:/ / doi.org/10.13053/cys-20-4-2506.

Martí-Sánchez, M., y Roger-Monzó, V. (2018). La percepción social de la homeopatía en la prensa digital española: un análisis semántico. Panace, 18(47), 115-123. Recuperado de: https://dialnet.unirioja.es/servlet/ articulo?codigo $=6629817$.

McManus, D. (2017). Reiki is better than placebo and has broad potential as a complementary health therapy. Journal of Evidence-Based Complementary \& Alternative Medicine, 22(4), 1051-1057. https://doi. org/10.1177\%2F2156587217728644.

Moreno, C. (2001). Métodos para medir la biodiversidad. Oficina Regional de Ciencia y Tecnología para América
Latina y el Caribe, UNESCO/Sociedad Entomológica Aragonesa (SEA).

Moretti, L., y Basler, H. (2016). Introducción a las prácticas en salud basadas en la evidencia: decisiones clínicas fundadas en pruebas científicas. En L. Medrano y L. Moretti (comps.), Prácticas basadas en la evidencia: ciencia y profesión en el campo de la salud (pp. 7-18). Editorial Brujas.

Muis, K., Chevrier, M., y Singh, C. (2018). The role of epistemic emotions in personal epistemology and self-regulated learning. Educational Psychologist, 53(3), $1-20$.

Murillo-Godínez, G. (2019). Panaceas, medicinas alternativas y similares: el auge y triunfo de la pseudociencia médica. Medicina Interna de México, 35(1), 113-143. https://doi.org/10.24245/mim.v35i1.2233.

Nussbaum, E., Sinatra, G., y Poliquin, A. (2008). Role of epistemic beliefs and scientific argumentation in science learning. International Journal of Science Education, 30(15), 1977-1999. https://doi. org/10.1080/09500690701545919.

Ojeda, R. (2011). Estrategias de andamiaje cognitivo en un ambiente de aprendizaje colaborativo en línea y la solución de problemas en una tarea auténtica: prueba de un modelo. HETS Online Journal, 2(1), 4-42.

Olvera, L. (2017). Creencias epistemológicas sobre el conocimiento en internet en estudiantes en tres momentos distintos de la carrera de Psicología SUAyED [Tesis de Licenciatura]. FES Iztacala, Universidad Autónoma de México. Recuperado de: http://132.248.9.195/ptd2018/ agosto/0779250/Index.html.

Pérez, A., Contreras, E., y Martínez, K. (2020). Disposición a la práctica basada en evidencia. Enseñanza e Investigación en Psicología, 2(3), 328-337.

Perry, W. (1970). Forms of intellectual and ethical development in the college years: A scheme. Nueva York: Holt, Rinehart \& Winston.

Rauchfleisch, A. (2017). The public sphere as an essentially contested concept: A co-citation analysis of the last 20 years of public sphere research. Communication and the Public, 2(1), 3-18. https://doi. org/10.1177\%2F2057047317691054.

Sackett, D., Rosenberg, W., Gray, J., Haynes, R., y Richardson, W. (1996). Evidence based medicine: What it is and what it isn't. British Medical Journal, (312), 71-72. 
Shah, C. (2015). Collaborative information seeking: From 'what?' and 'why?' to 'how?' and 'so what?'. En Collaborative Information Seeking (pp. 3-16). Cham: Springer. https://doi.org/10.1007/978-3-319-18988-8_1.

Terrazas, S. (2016). Creencias epistemológicas: premisas constitutivas de perspectivas acerca del conocimiento. Revista Electrónica de Investigación Educativa, 18(3). Recuperado de: http://redie.uabc.mx/redie/article/view/752.

Tu, Y., Shih, M., y Tsai, C. (2008) Eight graders' web searching strategies and outcomes: The role of task types, web experiences and epistemological beliefs. Computers \& Education, 51(3), 1142-1153.

Yun-Jo, A. (2010). Scaffolding wiki-based, ill-structured problem solving in an online environment. Journal of Online Learning and Teacbing, 6(4), 723. Recuperado de: https://jolt.merlot.org/vol6no4/an_1210.pdf.

Zanotto, M., y Gaeta, M. L. (2018). Epistemología personal y aprendizaje en la formación de investigadores. Perfiles Educativos, 40(162), 160-176. https:// doi.org/10.22201/iisue.24486167e.2018.162.58757.

Cómo citar este artículo:

Meza Cano, J. M., y Salas García, V. E. (2021).Análisis de textos pseudocientíficos desde las creencias epistemológicas de estudiantes de psicología. IE Revista de Investigación Educativa de la REDIECH, 12, e1234. doi: 10.33010/ ie_rie_rediech.v12i0.1234. 